\title{
An inexpensive variable time period integrator for electromyographic signals
}

\author{
D. J. MADER and A. E. MARBLE \\ Nova Scotia Technical College, Halifax, Nova Scotia, Canada \\ and \\ JOHN L. REEVES* \\ Dalhousie University, Halifax, Nova Scotia, Canada
}

\begin{abstract}
It is frequently desirable for clinical researchers to have some means of precisely quantifying average levels of electromyographic signals over a series of finite time periods. This paper presents the design and development of an inexpensive variable time period integrator that can be adapted to most commercially available electrom yograph equipment. The prototype instrument was found to give precise readings in the order of $1 \%-2 \%$ accuracy when adjusted to specification.
\end{abstract}

Clinical and research practices often necessitate some means of precisely quantifying the average level of electromyographic signals over a series of finite time periods. Although variable time period integrators are commercially available and have been briefly described elsewhere (Budzynski, Stoyva, \& Adler, 1970; Leaf \& Gaarder, 1971), detailed schematics and construction theory have not been provided. Moreover, the costs of these instruments exceed the budgetary allotments of many clinical researchers. The current availability of inexpensive integrated circuit operational amplifiers with good performance characteristics provides feasible alternatives for researchers operating on limited fundings.

The present report describes the design and development of an inexpensive variable time period integrator capable of displaying period-by-period EMG levels with $1 \%-2 \%$ accuracy. The operational characteristics of the integrator are such that the final figure accumulated on the readout meter represents the average level of EMG activity, in microvolts, for that particular time period. Thus, EMG levels might be 10.50 , $9.75,8.25,7.50$, and $8.25 \mathrm{microV}$ for five consecutive time periods of a predetermined length.

Preliminary clinical observations suggested four selectable integration time periods, $5,10,30$, and $60 \mathrm{sec}$ in duration. After considering several circuits on the basis of cost and accuracy, we decided that the timing circuit should consist of a monostable (one-shot) multivibrator with a Fairchild UA741C operational amplifier as the active element.

The prototype instrument is currently used with the Biofeedback Technology BFT-401 feedback electromyograph, ${ }^{1}$ but it is readily adaptable to any commercially available electromyograph. The instrument

*Reprint requests should be sent to John L. Reeves, Psychological Services Centre, Dalhousie University, Halifax, Nova Scotia, Canada. is highly reliable, simple to operate, and inexpensive, costing under $\$ 50$ to construct.

\section{PRINCIPLES OF OPERATION}

The Fairchild UA741C operational amplifier (op amp) was chosen as the basic building block of the integrator (see Fig. 1). An op amp is so designed that its characteristics approach that of an ideal amplifier. These characteristics are: (a) open-loop gain, $\mathrm{A}$, is very large (i.e., $3 \times 10^{5}$ ); (b) $\mathrm{e}_{\mathrm{o}}=0$, when $\mathrm{e}_{1}=\mathrm{e}_{2} ;$ (c) input resistance is very large (i.e., 1 Megohm); and (d) output resistance is almost zero. In the integrator described in the present paper, the op amp is used in various modes, but the main configuration is that of the basic integrator shown in Fig. 2. Mathematical analysis of this cicuit reveals that the output voltage, $e_{o}$, is related to the input voltage, $e_{i}$, by:

$$
e_{o}=-\frac{1}{R_{1} C_{f}} \int_{o}^{T} e_{i} d t
$$

where $T$ is the in tegration period. In designing a practical integrator, however, the voltage, $e_{0}$, is not due entirely to $e_{i}$. Two factors-input offset voltage and input bias current-contribute to the voltage that appears at the output terminal and in part causes the total output voltage to be independent of $e_{i}$. The offset voltage at the input terminals is integrated, and causes the output to drift. The input bias current that flows through the feedback capacitor causes it to charge, creating a voltage at the output terminals which is independent of $e_{i}$.

Both of these factors require compensation in the design of a practical, useful integrator. The standard procedure for eliminating offset voltage is to use the null offset terminals provided on the op amp for that purpose. Several methods have been employed to eliminate or reduce the effect of input bias current. One 


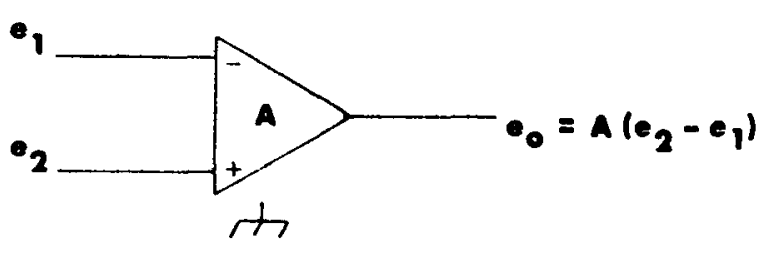

Fig. 1. Basic operational amplifier.

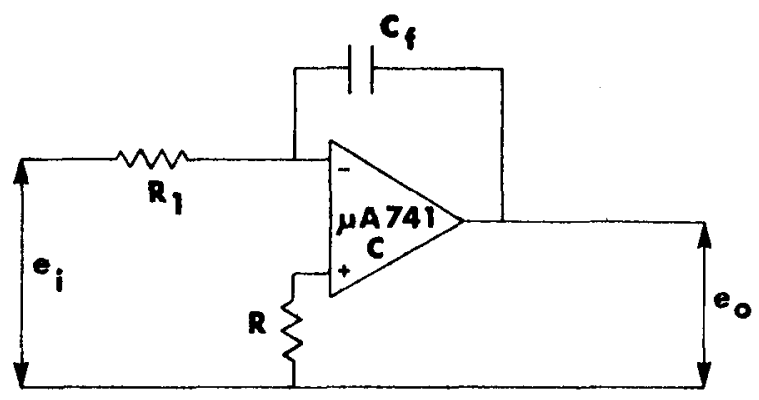

Fig. 2. Basic integrator.

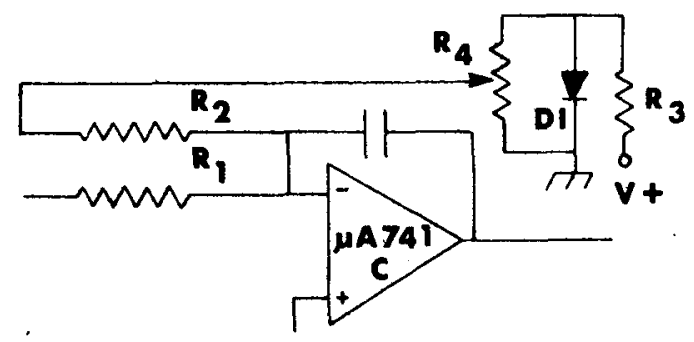

Fig. 3. Input bias reduction circuit.

device used to overcome this problem in long time-period integrators is the chopper stabilized operational amplifier. This op amp has long-term dc stability, but it is expensive (\$40) and it is not as readily available as a general-purpose UA 741 . The reduction of drift due to input bias current in the integrator described here is accomplished by supplying the input bias current to the op amp from an external source. This source is shown in Fig. 3, and is made up of a 9-V supply from the BFT-401 electromyograph. Diode $D_{1}$ is forward biased through $R_{3}$ so that $R_{4}$, a 10 -turn $10 \mathrm{~K}$ potentiometer, has a constant voltage of $0.43 \mathrm{~V}$ across it. Resistor $R_{2}$ is large (2.2 Megohm). Using this external source of bias current, the drift in output voltage, with no input signal $\left(e_{i}\right)$, after the offset voltage has been nulled, can be reduced to less than $5 \mathrm{mV} / \mathrm{min}$.

The integrator was designed to accept an input from the BFT-401 electromyograph, which has a sensitivity of $40 \mathrm{mV} / \mathrm{microV}$. Thus for $50 \mathrm{microV}$ of electromyographic signal input, the BFT -401 produces a $2-\mathrm{V}$ output. For convenience, the integrator was designed to integrate to a final value of $5 \mathrm{~V}$, when the average level of the EMG signal was 50 microV. Four time periods of integration were built into the integrator by using a one-shot multivibrator as a timing circuit, as shown in Fig. 4. In Fig. 4, the op amp, $Q_{1}$, is the active element of the one-shot multivibrator. Initially, the voltage on the output terminal of the one-shot is $+7 \mathrm{~V}$. The output voltage of $Q_{2}$ is zero, since it is a summing amplifier and is adding the $+7 \mathrm{~V}$ from the one-shot to $-7 \mathrm{~V}$ from the voltage divider. When the start button is pressed, $-9 \mathrm{~V}$ is applied to the noninverting input of the op amp, $Q_{1}$, causing its output terminal to switch to $-7 \mathrm{~V} . \mathrm{Q}_{2}$ now becomes saturated, and its output voltage changes from 0 to $+7 \mathrm{~V}$, which is sufficient to operate the relay and in turn connects the EMG signal to the integrator input.

The time during which the EMG signal is applied to the integrator input terminal is determined by $\mathrm{C}_{2}$ and $R_{5}$. When the output terminal of the one-shot switches to $-7 \mathrm{~V}$, the capacitor $\mathrm{C}_{2}$ starts to charge through the series combination of $1 \mathrm{Kohm}$ and $\mathrm{R}_{5} \cdot \mathrm{C}_{2}$ charges until the voltage applied to the inverting input terminal is slightly larger than the voltage at the noninverting input. At this moment, the one-shot switches back to the initial state of $+7 \mathrm{~V}$ on its output terminal. Four values of $R_{5}$ were selected in order to obtain four integration times of $5,10,30$, and $60 \mathrm{sec}$. The overall integrator circuit is shown in Fig. 5. All resistors are 5\% tolerance and have calibration 10-turn potentiometers to achieve precision settings. Also, a scale-shifting network has been introduced before the relay in order to provide accurate readings on low-level inputs (see SCALE in Fig. 5). A pushbutton switch is provided in order to reset the integrator after each reading. This switch has a second pole connected across $\mathrm{C}_{2}$ in the timing circuit to ensure that the reset cycle of the timer will be completed when a new reading is started. A two-position switch, $S_{3}$, connects a dc microammeter to the output of the integrator. In Position A, the meter reads directly in

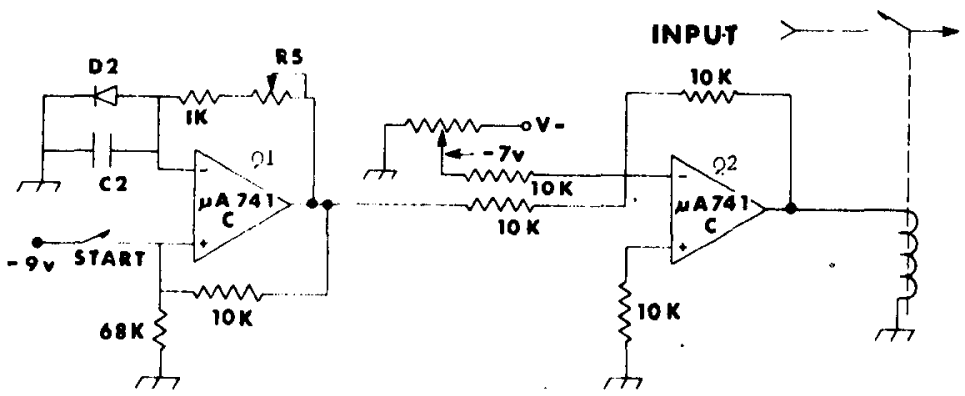

Fig. 4. One-shot multivibrator timing circuit. 


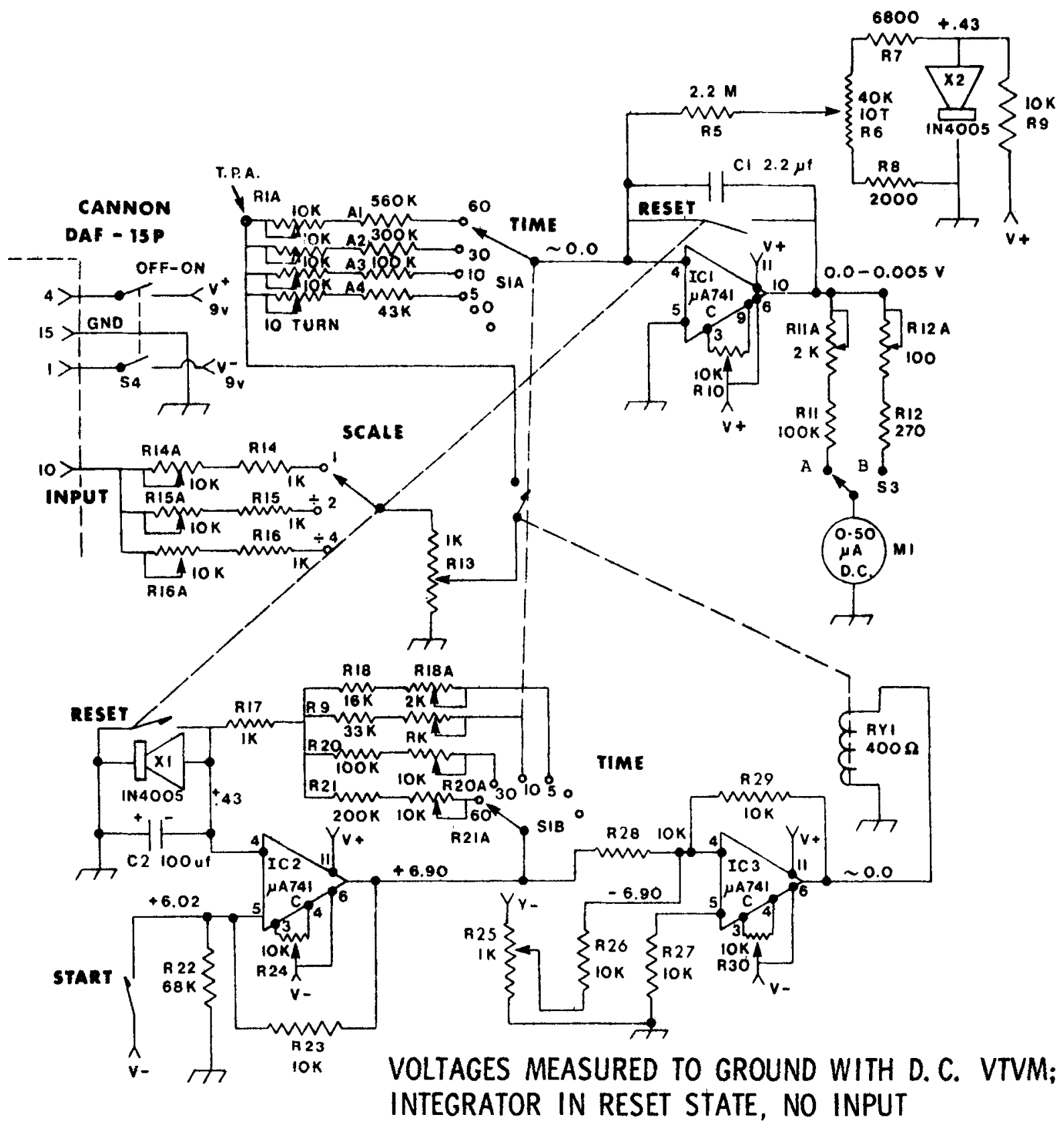

Fig. 5. Overall integrator circuit.

average microvolts at the pickup electrodes. In Position $\mathrm{B}$, the meter reads $100 \mathrm{mV}$ full scale, and is used to adjust the integrator drift to a minimum.

\section{ALIGNMENT INSTRUCTIONS}

\section{Null Offset}

The null offset adjustment is not critical for IC2 and IC3 and may be adjusted to the approximate center of the null offset potentiometer.

The null offset for the integrator should be adjusted by shorting Pin 4 of IC1 to ground, holding the reset button in and adjusting R10 for zero voltage at Pin 10 .
Timing Interval

Connect a signal level triggered interval counter to T.P.A. (see schematic), connect a $2-\mathrm{V} 100-\mathrm{Hz}$ signal source to the input. Adjust the interval timer trigger threshold so that the instrument counts when RY1 is closed. Initiate the integrator timer and adjust R18A through R21A for 5-, 10-, 30-, and 60-sec intervals, as indicated by the interval timer.

\section{Meter Calibration}

Turn Switch S3 to the norm position. Connect a dc VTVM to Pin 10 of IC 1 . Apply a voltage of $1 \mathrm{~V} \mathrm{dc}$ at the integrator input. Set the timer switch, S1, at $5 \mathrm{sec}$. 


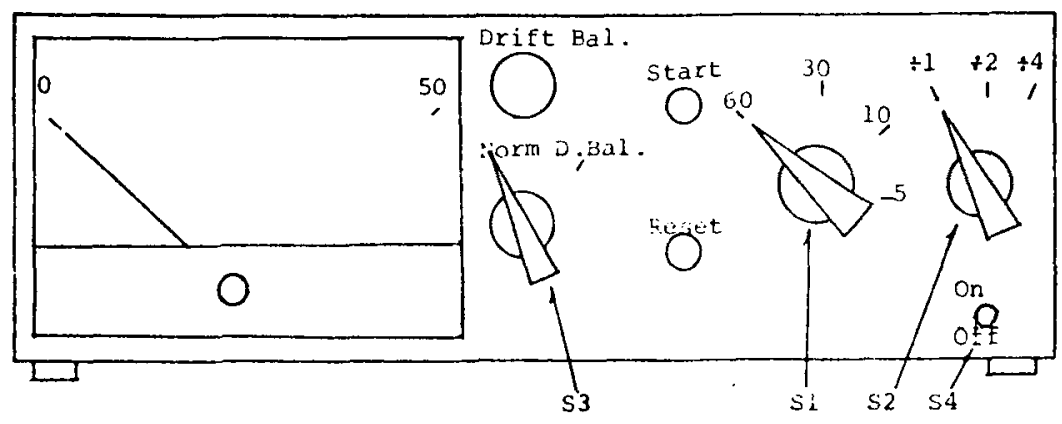

Fig. 6. Front panel controls.

Initiate an integration and allow the integrator to run up to some voltage. Adjust the calibration resistor, R11A, so that $M 1$ reads the proper voltage as indicated on the VTVM.

\section{Scale Factor Calibration}

Connect a dc VTVM to the wiper of R13. Adjust R14A to max resistance. Turn R14A back $1 / 8$ turn. Apply a voltage of $2.4 \mathrm{~V} \mathrm{dc}$, using a voltage calibrator, to the integrator input. Adjust R13 for a voltage of $0.1 \mathrm{~V}$ dc on the VTVM with $\mathrm{S} 2$ in the " 1 " position. With S2 in the " $\div 2$ " position, adjust R15A for $0.2 \mathrm{~V}$ on the VTVM. With S2 in the " $\div 4$ " position, adjust R16A for $0.4 \mathrm{~V}$ on the VTVM.

\section{Integrator Stage Gain Calibration}

Apply a voltage of $2.00 \mathrm{~V} \mathrm{dc}$ to the integrator input using a voltage calibrator. Set S1 to $60 \mathrm{sec}$. Press reset, then start, and when the integration stops adjust R1A lower in resistance if the meter reading is less than $50 \mathrm{mV}$ and higher in resistance if the reading is greater than $50 \mathrm{mV}$. Repeat the process until an integration gives a final reading of $50 \mathrm{mV}$. Similarly, adjust R2A, R3A, and R4A for the 30-, 10-, and 5-sec settings of S1.

\section{FRONT PANEL CONTROLS}

Figure 6 depicts the front panel of the variable time period integrator. The following provides an outline of the switches and their functions: (1) S4-When in the "on" position, power is connected to the integrator from the BFT-401 internal $\pm 9 . \mathrm{V}$ supply. (2) S3-Selects the meter scale; "Norm," normal integrate mode; "D. Bal," selects a sensitive scale for accurate adjustment of the drift balance control. (3) Drift balance-Adjusts the input bias current supply to the OP-AMP IC1. (4) Start-Begins the integration period subject to the setting of S1. (5) Reset-Resets the meter and internal timer after a reading has been taken. (6) Time (S1)-Selects the time period of integration, 5, 10,30, or $60 \mathrm{sec}$. (7) Scale (S2)-Selects the scale expansion factor (divide scale reading by 1,2 , or 4 , depending on setting).

\section{OPERATING INSTRUCTIONS}

(1) Select the "Norm" mode with Switch S3. (2) Turn the power switch, S4, "ON." (3) Wait 3-5 min for stabilization before proceeding, for best accuracy. (4) Turn the meter switch, S3, to the "D. Bal" position. (5) Push the reset button; release; and adjust the "Drift Bal" control for $0-0$ movement. If the reading goes off scale before this condition is achieved, push and release the reset button again and proceed with the adjustment. Note: If this adjustment has been done once, then it should require little future adjustment. (6) Turn the meter switch to the "Norm" position (IMPORTANT!); failure to do this may damage meter if an integration is attempted. (7) Select time period with $S 1-5,10,30$, or $60 \mathrm{sec}$. Note: If one of the two unused positions is selected, the integration period is infinite. (8) Select the desired scale factor using S2. For low EMG activity S2 $(\leqslant 10$ microV), use the $\div 4$ position. For $S 2$ with EMG $>10$ microV but $<25$ microV, use the $\div 2$ position. For higher activity, use the "1" position. The final meter reading must be modified as follows:

$\begin{array}{cc}\text { Position } & \text { Divide By } \\ \div 1 & 1 \\ \div 2 & 2 \\ \div 4 & 4\end{array}$

(9) When ready to take a reading, push the reset button and release it; to initiate the integration, push and release the start button. The meter needle will begin to rise gradually and stop at the end of the selected time period. This final reading, when modified by a scale factor (see Item 8), gives the average level of EMG activity (microvolts) at the input to the BFT-401 electromyograph. Note: If the settings of $\mathrm{S} 1$ or $\mathrm{S} 2$ are altered during an integration, the results will be meaningless. If the reset button is pushed before the end of the integration period, the period will be reinitiated. (10) When the reading has been noted, another may be taken by again pushing the reset button and then the start button. (11) Periodically check the "Drift Bal" setting and readjust it if necessary.

\section{PERFORMANCE EVALUATION}

Performance evaluations indicated that calibration on the different scales could be adjusted to within $1 \%$ of 
the expected values. Moreover, the calibration was found to be stable over long durations of time. Such findings were to be expected, since the circuit experiences little temperature rise during long operation periods and the 10-turn calibration potentiometers used were of high precision.

The drift balance control could be adjusted, with a little practice, to give less than $5 \mathrm{mV}$ of drift over a $1-\min$ period. For a full-scale level of $5 \mathrm{~V}$, this corresponds to a drift error of less than $0.1 \%$ on all time scales.

The dc microammeter is a wide-scale type having a manufacturer's claimed accuracy of $1 \%-2 \%$, and is easily readable to three significant figures.

The low construction cost of the instrument resulted in certain disadvantages. For example, the manual operation of the device requires that the $\mathrm{E}$ be present to engage the "start" and "reset" buttons as well as "to record the score for each integration period throughout the duration of an experimental session. While we have found this advantageous in certain situations, such a procedure is a nuisance in others, i.e., with short integration periods of 5 and $10 \mathrm{sec}$ and/or over lengthy experimental sessions. One more costly solution to this problem would be the addition of a constant reset level circuit (Budzynski et al, 1970; Shaw, 1967). At the end of the integration period, this technique would hold the accumulated score on the readout meter for a preset intertrial interval, then reset itself and initiate a new integration trial, continuously resetting and integrating until the experimental session was over. Moreover, by displaying the output of the integrator on a strip-chart recorder, minimal time expenditure would be required of the $\mathrm{E}$.

\section{REFERENCES}

Budzynski, T. H., Stoyva, J. M., \& Adler, C. Feedback-induced muscle relaxation: Application to tension headaches. Journal of Behavior Therapy \& Experimental Psychiatry, 1970, 1, 205-211.

Leaf, W. B., \& Gaarder, K. R. A simplified electromyographic feedback apparatus for relaxation training. Journal of Behavior Therapy \& Experimental Psychiatry, 1971, 2, 39-43.

Shaw, J. C. Integration technique. In P. H. Venables and I. Martin (Eds.), Manual of psychophysiological methods. New York: Wiley, 1967. Pp. 403-465.

\section{NOTE}

1. Commercial EMG feedback system available through Bio-Feedback Technology, Inc., 10402 Trask Avenue, Garden Grove, California 92643.

\footnotetext{
(Received for publication January 17, 1974;
} revision received April 1, 1974.) 